\title{
Genome-wide comparative analysis revealed significant transcriptome changes in mice after Toxoplasma gondii infection
}

\author{
Boyin Jia ${ }^{1}$, Huijun Lu', Quan Liư ${ }^{3}$, Jigang Yin ${ }^{1}$, Ning Jiang ${ }^{1 *}$ and Qijun Chen ${ }^{1,2^{*}}$
}

\begin{abstract}
Background: Toxoplasma gondii is an intracellular parasite that can modulate host responses and presumably host behavior. Host responses as well as pathogenesis vary depending on the parasite strains that are responsible for infection. In immune competent individuals, T. gondii preferentially infects tissues of the central nervous systems (CNS), which might be an additional factor in certain psychiatric disorders. While in immune-compromised individuals and pregnant women, the parasite can cause life-threatening infections. With the availability of the genome-wide investigation platform, the global responses in gene expression of the host after T. gondii infection can be systematically investigated.

Methods: Total RNA of brain tissues and peripheral lymphocytes of BALB/C mice infected with RH and ME 49 strain T. gondii as well as that of healthy mice were purified and converted to CRNA with incorporated Cy5-CTP (experimental samples), or Cy3-CTP (control samples). The labeled cRNA probes were hybridized to the Whole Mouse Genome Microarray. The impact of parasite infection on gene expression in both brain tissues and peripheral lymphocytes were analyzed. Differentially expressed genes were revalidated with real-time quantitative reverse transcriptase-polymerase chain reaction (Q-PCR).
\end{abstract}

Results: Data indicated that the genes associated with immunity were up-regulated after infection by the two parasite strains, but significant up-regulation was observed in both brain tissues and peripheral lymphocytes of mice infected with ME49 strain compared to that infected by RH strain. The pathways related to pathogenesis of the nervous system were more significantly up-regulated in mice infected with RH strain.

Conclusions: Genetically distinct T. gondii strains showed clear differences in modulation of host pathophysiological and immunological responses in both brain tissue and peripheral lymphocytes. It was likely that some of the host responses to $T$. gondii infection were universal, but the immune response and CNS reaction were in a strain-specific manner.

Keywords: Toxoplasma gondii, RH strain, ME49 strain, Microarray, Gene ontology and pathway analysis, Brain tissues, Peripheral lymphocytes

\footnotetext{
* Correspondence: jiangning@jlu.edu.cn; cqj@jlu.edu.cn

${ }^{1}$ Key Laboratory of Zoonosis, Institute of Zoonosis/College of Veterinary Medicine, Jilin University, Xi An Da Lu 5333, Changchun 130062, China ${ }^{2} \mathrm{MOH}$ Key Laboratory of Systems Biology of Pathogens, Institute of Pathogen Biology, Chinese Academy of Medical Sciences \& Peking Union Medical College, Beijing, Dongdan Santiao 9, Beijing 100730, China

Full list of author information is available at the end of the article
} 


\section{Background}

Toxoplasma gondii is an obligatory intracellular parasite that causes diverse pathological effects in humans and other warm-blooded vertebrates $[1,2]$. T. gondii has an unusual clonal population structure which exists with limited genetic diversity but belongs to three distinct clonal lineages and displays markedly different levels of virulence in mice [3]. Type I strain, such as RH strain is considered as the most virulent in mice, and it has been frequently found in individuals at risk of atypical ocular toxoplasmosis [4]. Type II strain, like ME49 strain is less pathogenic with lower LD50 values than that of $\mathrm{RH}$ strain and this strain has been found in the majority of human infections [5]. Type III T. gondii is rarely found in humans, but the reason is unclear [6]. Approximately $80 \%$ of individuals are asymptomatic after $T$. gondii infection, partially due to effective innate responses [7]. T. gondii establishes parasitization in the host by crossing biological barriers such as gut epithelia, the placenta or the bloodbrain barrier [8]. Studies have found that parasites of type I strains grow faster in vitro than that of type II or III strains [9].

Parasite specific CD4+ and CD8+ T cell-mediated immunity, a strong Th1-type response which is predominant in the immunocompetent host's immune reaction, has been regarded as the main component in antiToxoplasma infection [10], meanwhile humoral immunity plays a supplementary role [11]. The combination of IL-12 and IFN- $\gamma$ was proposed as the core of defence against the parasite in early infection [12]. Neutrophils, macrophages, NK cells and NKT cells are another elements involved in the immune response to T. gondii infection [13]. However, once the host's immune system balance is broken, it would lead to serious consequences, such as in AIDS patients [14]. Missing or defectiveness in function of immune system will result in a large number of proliferation of tachyzoites, which will lead to a severe consequence to the host [12].

Microarray represents the first generation of analytical tools with the capacity of global gene expression profiling in both pathogen development and host-pathogen interactions [15]. It has been extensively used to identify alterations in gene expression of bacterial [16] and viral infection [17]. Microarray has also been used to investigate gene expression of parasites such as Eimeria maxima and Plasmodium falciparum [18,19]. In studies on T. gondii infections, microarray has been mainly applied to analyze cell-specific responses with cultivated cells such as human foreskin fibroblasts, macrophages, dendritic cells, Muller cells, rat retinal vascular endothelial cells [20-23]. Using a cDNA microarray, Fouts et al. compared the expression profiles of human foreskin fibroblast cells infected by bradyzoites and tachyzoites. It was found that more genes were up-regulated in the fast growing tachyzoite-infected cells [24]. Further studies with cultivated Muller cells have found that T. gondii could induce cell immune responses, which could not control the differentiation of the parasites [22]. In addition, Xiao et al. examined the transcriptional profile of human neuroepithelioma cells in response to infection of type I, II, III of $T$. gondii by using microarray analysis [25]. However, all these studies were performed with cell lines infected by $T$. gondii, whereas little is known about changes in hosts in vivo. Furthermore, several studies have indicated that parasite infection in the host neural cells may cause behavioral alterations, but no conclusive evidence has been identified [26-28]. More studies are still necessary in order to increase our understanding of host-parasite interactions in T. gondii infection. In this study, we systematically analyzed the transcriptomes in both brain tissues and peripheral lymphocytes in BALB/C mice after infection by Type I (RH strain) and II (ME49 strain) T. gondii respectively. We found that hosts displayed distinct neurological and immunological responses to the infection of the two strains, which may explain the pathological background of infection caused by genetically different $T$. gondii strains.

\section{Methods}

\section{Parasites and mouse tissues}

$\mathrm{RH}$ and ME49 strains of T. gondii were routinely kept in the laboratory by cell cultivation. Thirty female BALB/C mice (20-25 g) aged six to eight weeks were randomly divided into 3 groups. For collecting brain tissues and peripheral lymphocytes from infected mice, two groups of $\mathrm{BALB} / \mathrm{C}$ mice (10 per group) were peritoneally infected with $\mathrm{RH}$ and ME49 strain with 10 tachyzoites per mouse respectively. Infection was confirmed by Giemsa staining of peritoneal fluid and PCR [29,30]. Brain tissues were collected from infected mice 8 days after infection. At first, the infected mice were exsanguinated and the blood was mixed with heparin for separation of peripheral lymphocytes. The mice were then soaked in 75\% ethanol for 30 seconds. Whole brain tissues were harvested, rinsed extensively in PBS, and immediately frozen in liquid nitrogen.

Peripheral lymphocytes were purified using Lymphocyte Separation Medium (Lonza, NJ, USA). Briefly, the anticoagulated blood diluted $2 \times$ with Hank's solution was carefully layed over the lymphocyte separation solution. Samples were centrifuged at $1500 \mathrm{rpm} / \mathrm{min}$ for 15 minutes at room temperature. The lymphocytes concentrated in the interphase (white layer) between the plasma and the separation solution were collected and washed with Hanks solution 3 times before further processing. The use of laboratory animals was reviewed and approved by the Ethical Committee of Jilin University, Changchun, China. 


\section{RNA purification and microarray hybridization}

Total RNA of brain tissues and peripheral lymphocytes from mice infected with RH and ME 49 strain $T$. gondii, as well as of healthy mice, was extracted using the Trizol reagent (Invitrogen, CA, USA) according to the manufacturer's instruction. Purity and integrity of the RNA samples were assessed on the Agilent 2100 bioanalyzer with the RNA 6000 Nano LabChip reagent set (Agilent Technologies, China) as described [31]. The RNA was quantified spectrophotometrically and stored at $-80^{\circ} \mathrm{C}$ before use.

Before microarray hybridization, two batches of total RNA were labeled, and hybridized to Agilent Whole Mouse Genome $(4 \times 44 \mathrm{~K})$ Microarrays (one-color platform) (Agilent Technologies, Shanghai, China), which was comprised of 41,534 60-mer oligonucleotide probes representing over 41,000 mouse genes and transcripts. The array was designed to truly represent all known genes in the mouse genome and their resulting transcripts. Briefly, first strand cDNA was synthesized with a reverse transcription primer containing a T7 promoter sequence. cRNA was generated with T7 RNA polymerase, which simultaneously amplifies the target and incorporates Cy3- or Cy5-labeled CTP with at least a 100-fold RNA amplification rate. cRNA from of infected mice was labeled with Cy5-CTP, while cRNA from control samples was labeled with Cy3-CTP. The labeled cRNA samples were then fragmented in fragmentation buffer at $60^{\circ} \mathrm{C}$ for $30 \mathrm{~min}$ before hybridization. The slides were washed, stabilized and scanned with the Agilent Technologies Microarray Scanner (Agilent Technologies, Shanghai, China).

\section{Data acquisition and bioinformatic analysis}

Signals from all hybridization reactions were acquired and quality-checked by filtration of unspecific signals. Eventually, only signals with an absolute value of a Log2 Red/Green Lowess Normalized Ratio were selected and further analyzed. Data from six subject pairs were acquired. We used Gene ontology analysis and Pathway analysis to identify which of these genes showed significantly differential expression.

Gene ontology analysis was applied to annotate the main function of the differentially expressed genes according to the key functional classification of genes in NCBI $[32,33]$. The main categories of Gene ontology were involved in the top biological functions: molecular and cellular functions, diseases and disorders, and physiological system development and function [34]. Generally, Fisher's exact test and $\chi^{2}$ test were used to classify the Gene ontology category, and the false discovery rate (FDR) was calculated to correct the $\mathrm{p}$-value. The smaller the FDR, the less possibility of the error in judging the p-value [35]. Similarly, pathway analysis was used to find out the significant pathways of the differentially expressed genes according to KEGG [36]. The ratio of genes from the data set that map to the pathway divided by the total number of genes that map to the standard pathway was displayed. Fischer's exact test and $\chi^{2}$ test were used to calculate the corresponding p-value [37]. P-value $<0.05$ and FDR $<0.05$ were used as a threshold to select significant Gene ontology categories and Pathways [38].

\section{Data validation by quantitative real-time PCR}

Genes with significant differences in expression in mice before and after infection by $T$. gondii identified in microarray were further validated with Q-PCR. The RNA templates were reverse transcribed to cDNA using 200 U AMV Reverse Transcriptase (Promega, CA, USA) according to the manufacturer's instructions. Primers were designed to amplify sequences of 90-200 base pairs (bp) (Table 1). The $25 \mu \mathrm{l}$ R reaction contained $2 \mu \mathrm{g}$ total RNA, $0.5 \mathrm{mM}$ dNTP mix (Takara, Dalian, China), 200 U AMV Reverse Transcriptase, 40 U RNase inhibitor (Promega). Q-PCR reaction was performed using a 7500 Real-Time PCR System (Applied Biosystems, CA, USA) with SybrGreen PCR Master Mix (Applied Biosystems) according to the manufacturer's instructions. The genes encoding GAPDH (glyceraldehyde-3phosphate dehydrogenase) and $\beta$-actin were chosen as endogenous references. Relative expression was calculated using the comparative Ct method [39].

\section{Results}

Significant transcriptomic changes in mice infected by $\mathrm{RH}$ and ME49 strain T. gondii

Discriminant analysis demonstrated significant changes in the transcriptome of brain tissue and peripheral lymphocytes of mice before and after T. gondii infection of RH and ME49 strain (Table 2). Of the 41,174 genes presented in the mouse genome microarray, we identified 1,537 up-regulated and 1,213 down-regulated genes in brain tissues (Additional file 1), and 3,602 upregulated and 4,045 down-regulated genes in peripheral lymphocytes in the group of mice as a result of infection by $T$. gondii $\mathrm{RH}$ strain, compared to healthy control samples (Additional file 2) $(\mathrm{p} \leq 0.05)$. Similarly, we identified 1,842 up-regulated and 1,368 down-regulated genes in brain samples (Additional file 3) and 3,109 upregulated and 2,777 down-regulated genes in peripheral lymphocytes in ME49 strain infected mice compared to healthy controls (Additional file 4). Furthermore, 1,179 up-regulated and 1,613 down-regulated genes in the brain tissue (Additional file 5), and 1, 605 up-regulated and 1, 417 down-regulated genes in peripheral lymphocytes (Additional file 6), were identified in mice infected 
Table 1 Primers for selected genes analyzed using Q-PCR

\begin{tabular}{|c|c|c|c|}
\hline Genes & Primer sequence $\left(5^{\prime}-3^{\prime}\right)$ & GenBank number & Length of PCR products (bp) \\
\hline \multirow[t]{2}{*}{ Gnal } & TGGGCAACAGCAGCAAGA & AC140336 & 131 \\
\hline & $\overline{\text { AAGCAGCAGGCGGTGAGT }}$ & & \\
\hline \multirow[t]{2}{*}{$\mid \operatorname{tgb} 1$} & CTGGTCCCGACATCATCC & AC156608 & 81 \\
\hline & TCCAAATCAGCAGCAAGG & & \\
\hline \multirow[t]{2}{*}{ C3 } & TGGACCAGACCGAACAGT & BC043338 & 125 \\
\hline & $\overline{\text { GAAGGCAGCATAGGCAGA }}$ & & \\
\hline \multirow[t]{2}{*}{ Nos2 } & GAGCGAGTTGTGGATTGTC & AL592185 & 133 \\
\hline & $\overline{\text { CCAGGAAGTAGGTGAGGG }}$ & & \\
\hline \multirow[t]{2}{*}{ Fgfr2 } & TCAGCTGGGGGCGCTTCATC & AC157606 & 158 \\
\hline & $\overline{\text { GGGGGCAACCACGTACGCTTC }}$ & & \\
\hline \multirow[t]{2}{*}{ Fos } & AGACCGTGTCAGGAGGCA & BC029814 & 111 \\
\hline & CCATCTTATTCCGTTCCCT & & \\
\hline \multirow[t]{2}{*}{ Erbb4 } & ATGGCAGGTGCTATGGAC & AC167658 & 168 \\
\hline & GAAAGGTGGTTGGATTGT & & \\
\hline \multirow[t]{2}{*}{ Stat5b } & TGCTCATCAACAAGCCAGAC & AL591466 & 121 \\
\hline & $\overline{\text { AAAGGCATCAGATTCCAAA }}$ & & \\
\hline \multirow[t]{2}{*}{$\operatorname{lgf1r}$} & CCTTTGGGATGGTCTATGA & AC101879 & 88 \\
\hline & $\overline{\text { GCCTCGTTTACCGTCTTG }}$ & & \\
\hline \multirow[t]{2}{*}{ Rac2 } & TGATGGTGGACAGTAAGCC & AL590144 & 122 \\
\hline & TAGCGAGAAGCAGATGAGAA & & \\
\hline \multirow[t]{2}{*}{$\operatorname{ltgb2}$} & TCGGCTITGGGTCGTITG & AC153830 & 151 \\
\hline & TGGTTGGAGTTGTCGGTTA & & \\
\hline \multirow[t]{2}{*}{$\mathrm{Kdr}$} & GCAAATACAACCCTTCA & BC020530 & 95 \\
\hline & ACCAATACCCTTTCCTCA & & \\
\hline \multirow[t]{2}{*}{ Cblb } & GCTCGGCTACAGAAATAC & AK147367 & 167 \\
\hline & TCCCTGCTACCATCAATC & & \\
\hline \multirow[t]{2}{*}{$\mathrm{Cd} 4$} & ACTCACCCTCAAGATACCC & AC142254 & 119 \\
\hline & GAGCCACTITCATCACCA & & \\
\hline \multirow[t]{2}{*}{$\mathrm{Cd} 8 \mathrm{a}$} & ATTGGACTTCGCCTGTG & AC160090 & 109 \\
\hline & CTTTCGGCTCCTGTGGT & & \\
\hline \multirow[t]{2}{*}{ Jun } & ACGCCAACCTCAGCAACTTC & AK159196 & 193 \\
\hline & $\overline{\text { GTCTGCGGCTCTTCCTTC }}$ & & \\
\hline \multirow[t]{2}{*}{ TNF } & GTGGAACTGGCAGAAGAGG & CR974444 & 94 \\
\hline & CAAGCAGGAATGAGAAGAGG & & \\
\hline \multirow[t]{2}{*}{ Mapk3 } & CGGATTGCTGACCCTGA & BC029712 & 111 \\
\hline & GGATTTGGTGTAGCCCTTG & & \\
\hline \multirow[t]{2}{*}{ Ep300 } & CCAAGCATAGGGAATCAA & AC102262 & 184 \\
\hline & GGTCAGCAGAAGGAGCAG & & \\
\hline \multirow[t]{2}{*}{ Prkca } & AGTGCCAAGTTTGCTGTT & AL645535 & 143 \\
\hline & GGTAGGGCTTCCGTATGT & & \\
\hline \multirow[t]{2}{*}{ GAPDH } & AGGTCGGTGTGAACGGATTTG & BC082592 & 123 \\
\hline & TGTAGACCATGTAGTTGAGGTCA & & \\
\hline \multirow[t]{2}{*}{$\beta$-actin } & CGTGAAAAGATGACCCAG & AC144818 & 167 \\
\hline & AGAGCATAGCCCTCGTAGA & & \\
\hline
\end{tabular}


Table 2 Number of genes showing up and down regulation in mice after infection by $T$. gondii

\begin{tabular}{lccccc}
\hline & \multicolumn{2}{c}{ Up-regulation } & & \multicolumn{2}{c}{ Down-regulation } \\
\cline { 2 - 3 } \cline { 6 - 7 } & Brain & Periphery & & Brain & Periphery \\
\hline RH strain VS Healthy & 1,537 & 3,602 & & 1,213 & 4,045 \\
ME49 strain VS Healthy & 1,842 & 3,109 & & 1,368 & 2,777 \\
RH strain VS ME49 strain & 1,179 & 1,605 & & 1,613 & 1,417 \\
\hline
\end{tabular}

with $T$. gondii $\mathrm{RH}$ strain compared to that infected with ME49 strain.

\section{Gene ontology and pathway analysis revealed distinct expression patterns in the brain tissues of mice infected by RH and ME49 strain T. gondii}

Genes differentially expressed in mice infected by $\mathrm{RH}$ and ME49 strain T. gondii were characterized with Gene ontology and pathway analysis to further categorize the functions. Comparing the transcriptome of mouse brains infected by RH strain $T$. gondii with that of healthy mice showed that genes involved in the response to stress were differentially expressed. However, the number of up-regulated genes was more than the number that were down-regulated, such as response to drug, response to hypoxia, response to ethanol and response to estradiol stimulus. Genes involved in immune responses including antigen processing and presentation of peptide antigen via MHC class I were over-presented among the upregulated genes (Additional file 7: Figure S1A). On the other hand, genes involved in cell proliferation and adhesion including positive regulation of cell proliferation and regulation of epithelial cell proliferation were less presented among the down-regulated genes (Additional file 7: Figure S1B). In addition, in the brain tissue of $\mathrm{RH}$ infected mice, genes involved in neurological pathways such as focal adhesion, Wnt signaling and insulin signaling were up-regulated. The down-regulated genes were related to the nervous system including neuroactive ligand-receptor interaction and Alzheimer's disease in the same group of mice (Figure $1 \mathrm{~A}$ and B). In addition to the signaling pathway mentioned above, there were 12 up-regulated genes and 8 down-regulated genes in toll-like receptor signaling pathways in the brain tissue of mice infected by RH strain $T$. gondii. Similarly, there were 13 up-regulated genes and 12 down-regulated genes in Jak-STAT signaling pathway (Figure 1A and B).

The analysis of the transcriptome of the brains in mice infected by ME49 strain T. gondii as compared with that of healthy mice, showed that the most primary changes were found in expression of genes involved in inflammatory and immune responses, such as innate immune responses, antigen processing and presentation, positive regulation of I-kappaB kinase/NF-kappa B cascade including NF-kappa B transcription factor activity (Additional file 7: Figure S1C). On the other hand, the primary Gene Ontology categories for down regulated genes were related to the response to stress, such as responses to drugs and ethanol, hypoxia; responses to stimulus of glucose, estradiol and estrogen, glucocorticoid, and responses to lipopolysaccharide (Additional file 7: Figure S1D). Thus, the responses to stress in the brain tissue of mice infected by ME49 strain were opposite to that in mice infected by RH strain.

Furthermore, in the brain tissue of mice infected by ME49 strain, differential expression in genes involved in immune pathways such as antigen processing and presentation, natural killer cell mediated cytotoxicity, $\mathrm{T}$ cell receptor signaling pathway, toll-like receptor signaling pathway and chemokine signaling pathway were more prominent. These pathways were differentially expressed and all of them were up-regulated in brain tissue of mice infected by ME49 strain T. gondii. In the genes involved in neurological pathways, 21 were up-regulated genes and 21 were down-regulated in neuroactive ligandreceptor interaction. In the genes involved in Jak-STAT signaling pathway, 21 genes were up-regulated and 12 genes were down-regulated (Figure $1 \mathrm{C}$ and $\mathrm{D}$ ).

Further analysis carried out by comparing the expression in genes involving pathways in the brain tissue infected by $\mathrm{RH}$ strain $T$. gondii versus that by ME49 strain; showed that the number of down-regulated pathways was more than the number up-regulated. The main difference was in the pathways related to immune responses in the brain. The pathways of chemokine signaling pathway, toll-like receptor signaling pathway, JakSTAT signaling pathway, B cell receptor signaling pathway and $\mathrm{T}$ cell receptor signaling pathway were all downregulated in the brain of $\mathrm{RH}$ strain infected mice compared to that of ME49 strain infected mice. On the other hand, the genes in pathways related to neurological responses such as focal adhesion, insulin signaling and neuroactive ligand-receptor interaction were more up-regulated in the brain tissue of $\mathrm{RH}$ strain infected mice compared to that of ME49 strain infected mice (Figure 1E and F).

\section{Gene ontology and pathway analysis revealed distinct expression patterns in the peripheral lymphocytes of mice infected by RH and M49 strain T. gondii}

We further analyzed the transcriptomic changes in the peripheral lymphocytes in mice infected by RH and ME49 strain T. gondii. The transcriptional changes in lymphocytes in the RH strain-infected mice compared to that of uninfected mice were similar to that of the brain tissues (Additional file 8: Figure S2A and S2B). The most prominent up-regulated genes were involved in olfactory transduction, ribosome, cell adhesion molecules (CAMs), hematopoietic cell lineage related to metabolism. Genes 


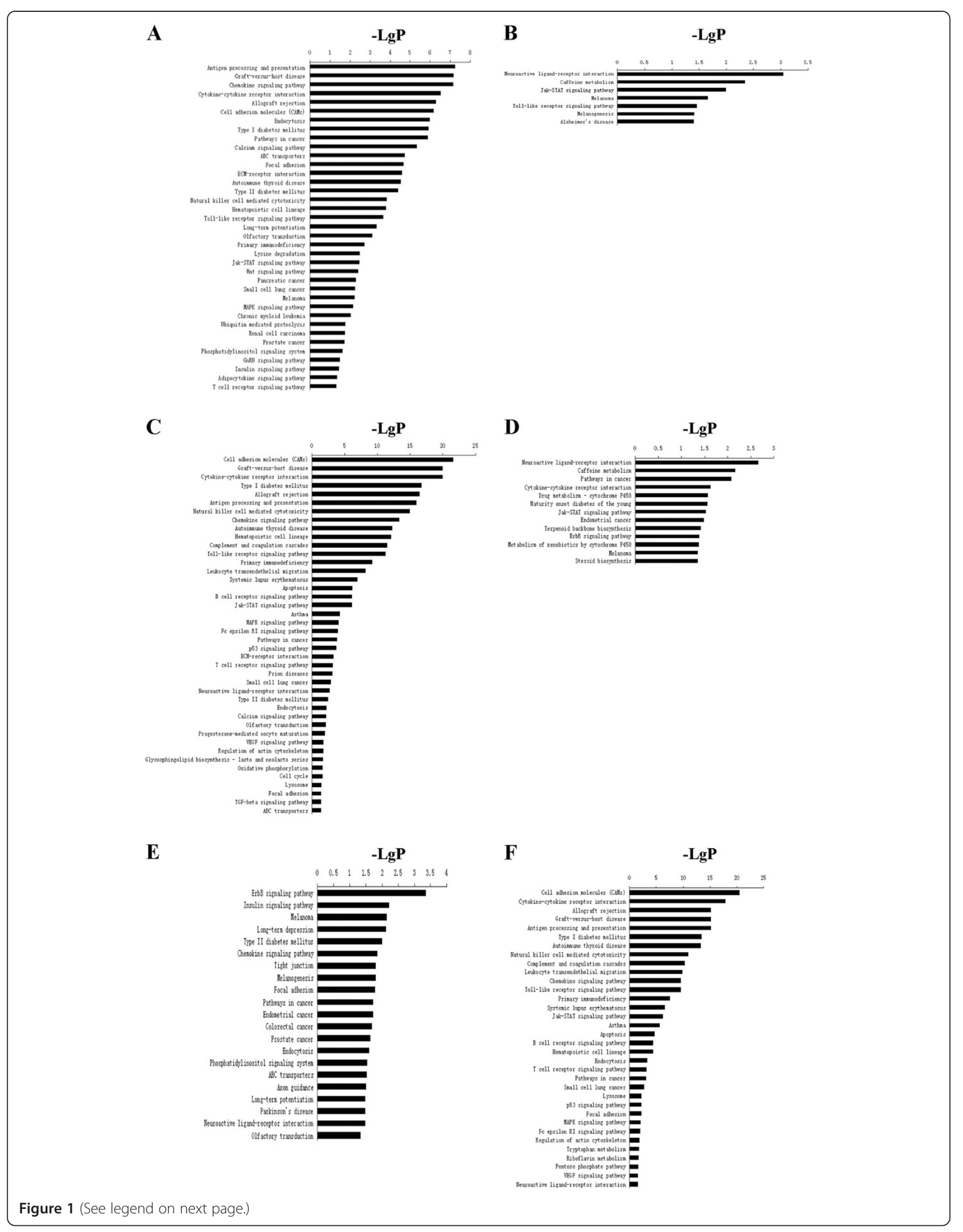


(See figure on previous page.)

Figure 1 KEGG pathway analysis for differentially expressed genes in the brain tissues of mice infected by RH and ME49 strain $T$.

gondii. (A and B) Pathways significantly up- and down-regulated in the brain tissues of mice infected by RH strain T. gondii compared to that of un-infected mice. (C and D) Pathways significantly up- and down-regulated in the brain tissues of mice infected by ME49 strain T. gondii compared to that of un-infected mice. (E and $\mathbf{F}$ ) Pathways significantly up- and down-regulated in the brain tissues of mice infected by RH strain T. gondii compared to that of ME49 strain infected mice. P-value $<0.05$ and FDR $<0.05$ were used as a threshold to select significant KEGG pathways.

involved in immune pathways such as toll-like receptor signaling pathways, chemokine signaling pathways, JakSTAT signaling pathways and natural killer cell mediated cytotoxicity were moderately up-regulated. On the other hand, the pathways such as $\mathrm{T}$ cell receptor signaling pathways, antigen processing and presentation, and B cell receptor signaling pathways were all down-regulated compared to those in the healthy mice. Additionally, pathways of oxidative phosphorylation, sugar and glycerolipid metabolism involved in energy metabolism were upregulated in the lymphocytes of $\mathrm{RH}$ strain-infected mice (Figure 2A and B).

Analysis in genes related to pathways in the peripheral lymphocytes in the ME49 strain T. gondii infected mice compared to those of healthy mice revealed that the number of significantly up-regulated pathways was more than those down-regulated, and the most up-regulated pathways were those involved in immune responses. In the peripheral lymphocytes in the ME49 strain T. gondii infected mice, the immune pathways such as cytokinecytokine receptor interaction, toll-like receptor signaling pathway, chemokine signaling pathway, Jak-STAT signaling pathway and natural killer cell mediated cytotoxicity were prominently up-regulated (Figure 2C and D). On the other hand, pathways such as $\mathrm{T}$ cell receptor signaling pathway, B cell receptor signaling pathway, antigen processing and presentation were down-regulated. In addition, the neuroactive ligand-receptor interaction pathway related to the nervous system was up-regulated compared to that of healthy mice (Figure $2 \mathrm{C}$ and $\mathrm{D}$ ).

However, the expression changes in the lymphocytes of mice infected by RH strain differed significantly from those infected by ME49 strain. The result of this analysis showed that the number of significantly up-regulated pathways was more than that of down-regulated pathways, and the main up-regulated pathways were those involved in metabolism, such as alpha-Linolenic acid metabolism, sulfur metabolism, metabolism of xenobiotics by cytochrome P450, vitamin B6 metabolism and so on. In addition to the signaling pathway, there were 14 up-regulated genes and 17 down-regulated genes in cell adhesion molecules (CAMs). Similarly, there were 24 up-regulated genes and 22 downregulated genes in neuroactive ligand-receptor interaction (Figure 2E and F).

\section{Differentially expressed genes were confirmed by Q-PCR}

Genes with significant differences in expression in mice before and after infection with $T$. gondii, that were identified in microarray were further validated with Q-PCR. Thirteen genes with up-regulation profiles and 10 genes with down-regulation profiles were selected (Table 1) and evaluated by Q-PCR. The results (Figure 3) confirmed the microarray data.

\section{Discussion}

Toxoplasma gondii is an obligate intracellular parasite that can infect all nucleated cells. The parasite can not only cause severe pathogenesis to the host, but also actively manipulates host responses to benefit its proliferation and dissemination. The outcome following $T$. gondii infection depends on various factors, including host immune status and parasite factors. T. gondii mainly resides in the neuron cells during chronic infection, which might cause certain psychiatric disorders in the immune competent host $[40,41]$. To better understand the pathophysiology of toxoplasmosis, the global gene expression profiles in brain and peripheral lymphocytes following $T$. gondii infection were systematically investigated with a genome microarray platform in this study. Instead of simply reporting a list of differentially expressed genes, we sought to study the immune and neurological implications of gene expression changes in the context of biological pathways.

Previous studies have indicated that $T$. gondii induces responsive changes in protein expression in the brain tissue of infected mice [42]. Here, the global expression patterns in the brain tissue after infection by different phenotypic $T$. gondii strains were analyzed. The main differences in gene expression in the brain tissues in mice infected by RH strain and ME49 strain were in the aspects of neurological damage and immune responses. The genes involved in pathways related to neurological responses such as focal adhesion, insulin signaling and neuroactive ligand-receptor interaction were more up-regulated in the brain tissue of $\mathrm{RH}$ strain infected mice compared to that of ME49 strain infected mice (Figure 1). Contrary to the up-regulation of a large number of pathways, Alzheimer's disease and neuroactive ligand-receptor interaction were the down-regulated pathways observed in the brain tissue of $\mathrm{RH}$ strain infected mice compared to that of 


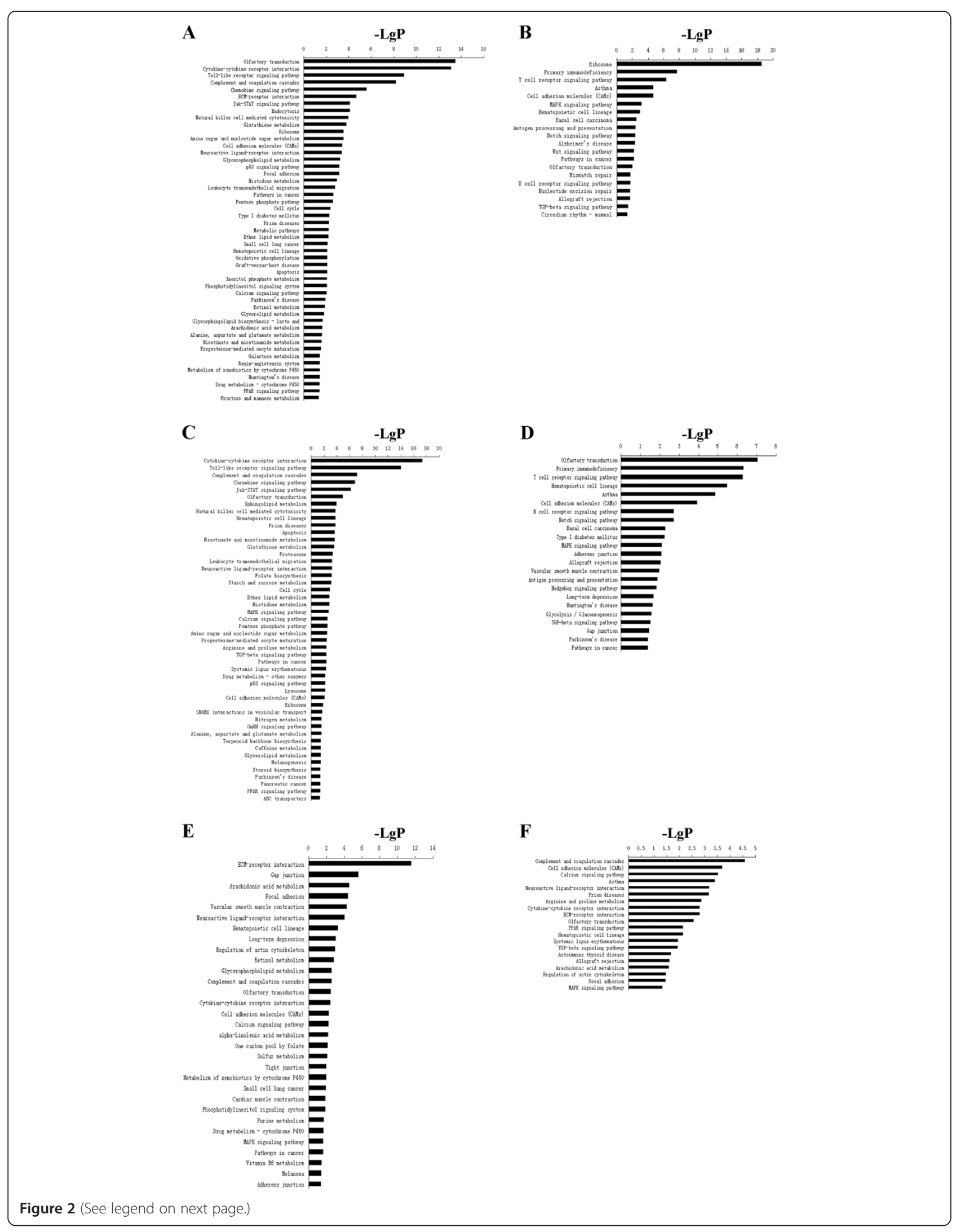


(See figure on previous page.)

Figure 2 KEGG pathway analysis for differentially expressed genes in KEGG pathway analysis for differentially expressed genes in peripheral lymphocytes of mice infected by RH and ME49 strain T. gondii. (A and B) Pathways significantly up- and down-regulated in peripheral lymphocytes of mice infected by RH strain T. gondii compared to that of un-infected mice. (C and D) Pathways significantly up- and down-regulated in peripheral lymphocytes of mice infected by ME49 strain T. gondii compared to that of un-infected mice. (E and $\mathbf{F})$ Pathways significantly up- and down-regulated in peripheral lymphocytes of mice infected by RH strain T. gondii compared to that of ME49 strain infected mice. P-value $<0.05$ and FDR $<0.05$ were used as a threshold to select significant KEGG pathways.

un-infected mice. The primary features of Alzheimer's disease $(\mathrm{AD})$ have previously been described as a neurodegenerative disorder exhibiting intracellular neurofibrillary tangles and extracellular senile plaques [43]. Previous serological studies proved that $T$. gondii infection may be an aetiological factor associated with $\mathrm{AD}$ [44]. Jung et al. reported that $T$. gondii caused learning and memory disorder in $\mathrm{Tg} 2576$ mice [45]. Down regulation of factors related to $\mathrm{AD}$ may reflect the ability of the parasite to inhibit cell apoptosis, which may maintain a better environment for the chronic parasitization in the host. Further, insulin signaling was another over-represented biological processes involved in neurological function. It was one of most important neurotrophic factors that seem to play a key role in the pathogenesis of $\mathrm{AD}$ [46]. For example, insulin signaling deficiency was related to AD pathology [47]. Thus, it is postulated that $\mathrm{RH}$ strain is prone to infect neuron cells and causes more brain damage than ME49 strain. Although the immune responses in the brains of mice infected by either RH or ME49 strain T. gondii were up-regulated (Figure 1), the genes involved in chemokine signaling pathway, tolllike receptor signaling pathway, Jak-STAT signaling pathway, B cell receptor signaling pathway and $\mathrm{T}$ cell receptor signaling pathway in the brain tissue of mice infected by
ME49 strain compared to that of RH strain infected mice were significantly more up-regulated. Earlier studies have shown that TLR11 and MyD88 were required in the activation of early innate immune responses and mice which lack MyD88 were highly susceptible to $T$. gondii infection $[48,49]$. In this study, MyD88 was found to be significantly up-regulated in the mice infected by ME49 strain compared to that infected by RH strain. This may explain the pathological difference between the mice infected by the two toxoplasmal strains. Furthermore, the activation of the T cell receptor contributed to cellular immunity, which was conducive to protective immunity of humans and mice [50]. In this study, $T$ cell receptor signaling pathway was found significantly up-regulated in the host infected with ME49 strain compared to that infected with $\mathrm{RH}$ strain. Thus it is likely that the innate and adaptive immune responses of the host were more strongly elicited by ME49 strain.

Peripheral lymphocytes are the most important immune components in responses to pathogen infection. However, the gene expression of immune cells during infections can be manipulated by the parasites. Here the transcriptomes of peripheral lymphocytes in mice infected by $\mathrm{RH}$ and ME49 strain were compared. The

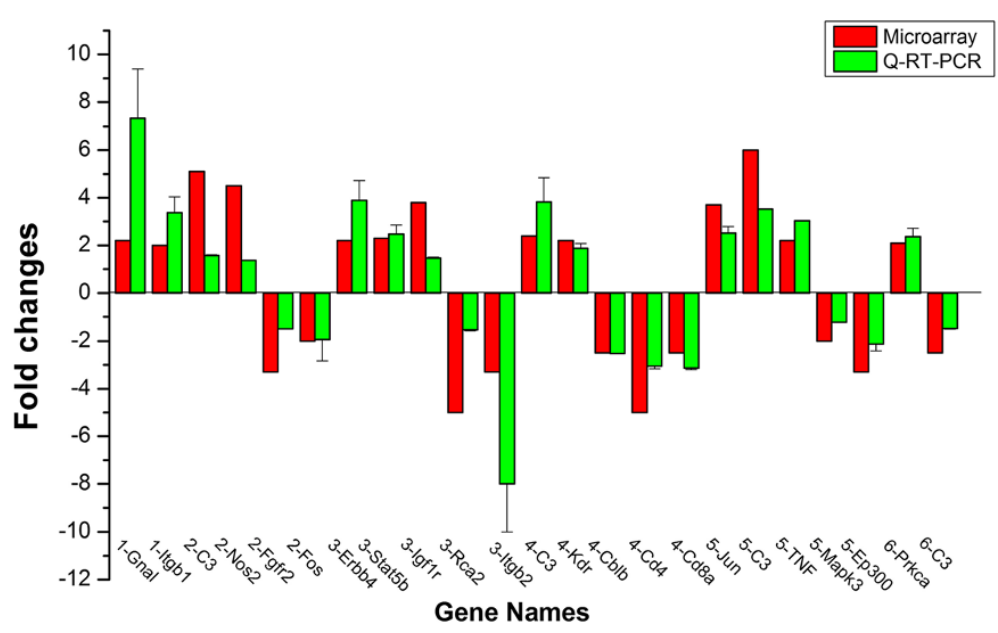

Figure 3 Validation of up- and down-regulated expressed genes by Q-PCR. The expression of 13 up-regulated genes such as Gnal, Itgb1, C3, Nos2, Erbb4, Stat5b, Igf1r, Kdr, Jun, TNF, Prkca and 10 down-regulated genes such as Fgfr2, Fos, Rca2, Itgb2, Cblb, Cd4, Cd8a, Mapk3, Ep300, C3 (C3 was regulated among ME49 strain brain versus control, RH strain peripheral lymphocytes versus control, ME49 strain peripheral lymphocytes versus control, RH strain peripheral lymphocytes versus ME49 strain peripheral lymphocytes) identified in microarray assay (in red color) was re-evaluated by Q-PCR (in green color). The Q-PCR results confirmed the data of the microarray. 
differences in gene expression in the peripheral lymphocytes in mice infected by RH strain and ME49 strain $T$. gondii were more significant than that in the brain tissues, especially with the genes involved in immune responses. In the peripheral lymphocytes in the ME49 strain $T$. gondii infected mice, the immune pathways such as cytokine-cytokine receptor interaction, toll-like receptor signaling pathway, chemokine signaling pathway, Jak-STAT signaling pathway and natural killer cell mediated cytotoxicity were prominently upregulated compared to those of $\mathrm{RH}$ strain infected mice (Figure 2). Furthermore, we observed that the energy metabolism pathways were significantly up-regulated in mice infected by $T$. gondii RH strain compared to those infected by ME49 strain. This may be a reflection of the difference in proliferation rate between the two parasite strains. The fast-growing RH strain would cause more energy consumption in the host, and earlier studies have shown that oxidative phosphorylation was essential for maintaining the ATP level for the growing tachyzoite [51]. Our results also confirm the earlier findings. Furthermore, Weilhammer et al. reported that host cell glycolysis could promote the growth of tachyzoite and inhibit bradyzoite conversion [52]. Our data revealed that Glycolysis/Gluconeogenesis was down-regulated in mice infected by T. gondii ME49 strain, but there were no other significant changes in the energy metabolism in the host. This may indicate that the parasites were transforming from tachyzoite to the bradyzoite phase.

\section{Conclusions}

The transcriptomic profiles of mice after infection by genetically distinct strains of $T$. gondii showed clear diversity in both brain tissues and peripheral lymphocytes. Gene ontology, pathway analysis indicated that the innate and adaptive response of the host was more strongly elicited in ME49 strain infected mice. Whereas, gene responses related to neurological disorders were prominent in RH strain $T$. gondii infected mice. With respect to the ability of $T$. gondii to influence host gene expression, it is likely that some of these effects were universal, while some of the genes involved in the immune response and CNS regulation of brain tissue and peripheral lymphocytes are strain-specific in manner. Future research should extend such studies to various types of immune response and brain cells between strains of different virulence effects on host gene expression.

\section{Additional files}

Additional file 1: Microarray hybridization results of up- and downregulated genes in mouse brain after infection by $\mathrm{RH}$ strain $T$. gondii.
Additional file 2: Microarray hybridization results of up- and downregulated genes in mouse peripheral lymphocytes after infection by $\mathrm{RH}$ strain $T$. gondii.

Additional file 3: Microarray hybridization results of up- and downregulated genes in mouse brain after infection by ME49 strain T. gondii. Additional file 4: Microarray hybridization results of up- and downregulated genes in mouse peripheral lymphocytes after infection by ME49 strain $T$. gondii.

Additional file 5: Microarray hybridization results of up- and downregulated genes in mouse brain tissues after infection by $\mathrm{RH}$ strain versus that infected by ME49 strain T. gondii.

Additional file 6: Microarray hybridization results of up- and downregulated genes in mouse peripheral lymphocytes after infection by RH strain versus that infected by ME49 strain $T$. gondii.

Additional file 7: Figure S1. GO category based on biological process for differentially expressed genes in brain tissues in mice infected by RH and ME49 strain T. gondii. (A and B) The significantly up- and downregulated genes in the brain tissues of mice infected by RH strain $T$. gondii compared to that of un-infected mice. (C and $\mathbf{D}$ ) The significantly up- and down-regulated genes in the brain tissues of mice infected by ME49 strain T. gondii compared to that of un-infected mice. $P$-value $<$ 0.05 and FDR $<0.05$ were used as a threshold to select significant GO categories.

Additional file 8: Figure S2. GO category based on biological process for differentially expressed genes in peripheral lymphocytes in mice infected by RH and ME49 strain T. gondii. (A and B) The significantly upand down-regulated genes in peripheral lymphocytes of mice infected by RH strain T. gondii compared to that of un-infected mice. (C and $\mathbf{D}$ ) The significantly up- and down-regulated genes in peripheral

lymphocytes of mice infected by ME49 strain T. gondii compared to that of un-infected mice. $P$-value $<0.05$ and FDR $<0.05$ were used as a threshold to select significant $\mathrm{GO}$ categories.

Competing interests

The authors declare that they have no competing interests.

\section{Authors' contributions}

$\mathrm{BJ}, \mathrm{QC}$ and NJ conceived and participated in the design of the platform; BJ, $\mathrm{HL}, \mathrm{QL}$ and $\mathrm{JY}$ carried out the experiments. The manuscript was drafted by BJ, QC and NJ. The final version was read and approved by all authors.

\section{Acknowledgement}

We appreciate the technical assistance from Shanghai Biochip Centre (SBC) for microarray hybridization and bioinformatic analysis. This study was supported by the national science \& technology specific projects (2008ZX10004-011).

\section{Author details}

${ }^{1}$ Key Laboratory of Zoonosis, Institute of Zoonosis/College of Veterinary Medicine, Jilin University, Xi An Da Lu 5333, Changchun 130062, China. ${ }^{2} \mathrm{MOH}$ Key Laboratory of Systems Biology of Pathogens, Institute of Pathogen Biology, Chinese Academy of Medical Sciences \& Peking Union Medical College, Beijing, Dongdan Santiao 9, Beijing 100730, China. ${ }^{3}$ Institute of Military Veterinary, Academy of Military Medical Sciences, Key Laboratory of Jilin Province for Zoonosis Prevention and Control, 666 Liuying Xilu, Changchun 130122, Jilin Province, China.

Received: 24 February 2013 Accepted: 19 May 2013

Published: 4 June 2013

References

1. Dubey J: Toxoplasma, Hammondia, Besnotia, Sarcosystis, and other tissue cyst-forming coccidia of man and animals. In Parasitic protozoa. Edited by Kreier JP. New York: Academic; 1977:101-237.

2. Dubey J, Beattie C: Toxoplasmosis of animals and man. Boca Raton, FL: CRC Press; 1988:220. 
3. Howe DK, Sibley LD: Toxoplasma gondii comprises three clonal lineages: correlation of parasite genotype with human disease. J Infect Dis 1995, 172:1561-1566.

4. Grigg ME, Ganatra J, Boothroyd JC, Margolis TP: Unusual abundance of atypical strains associated with human ocular toxoplasmosis. $J$ Infect Dis 2001, 184:633-639.

5. Ajzenberg D, Cogne N, Paris L, Bessieres MH, Thulliez P, Filisetti D, Pelloux $H$, Marty P, Darde ML: Genotype of 86 Toxoplasma gondii isolates associated with human congenital toxoplasmosis, and correlation with clinical findings. J Infect Dis 2002, 186:684-689.

6. Howe DK, Honore S, Derouin F, Sibley LD: Determination of genotypes of Toxoplasma gondii strains isolated from patients with toxoplasmosis. J Clin Microbiol 1997, 35:1411-1414.

7. Filisetti D, Candolfi E: Immune response to Toxoplasma gondii. Ann Ist Super Sanita 2004, 40:71-80.

8. Saeij JP, Boyle JP, Boothroyd JC: Differences among the three major strains of Toxoplasma gondii and their specific interactions with the infected host. Trends Parasitol 2005, 21:476-481.

9. Radke JR, Striepen B, Guerini MN, Jerome ME, Roos DS, White MW: Defining the cell cycle for the tachyzoite stage of Toxoplasma gondii. Mol Biochem Parasitol 2001, 115:165-175.

10. Yap GS, Sher A: Cell-mediated immunity to Toxoplasma gondii: initiation regulation and effector function. Immunobiology 1999, 201:240-247.

11. Kang H, Remington JS, Suzuki Y: Decreased resistance of B cell-deficient mice to infection with Toxoplasma gondii despite unimpaired expression of IFN-gamma, TNF-alpha, and inducible nitric oxide synthase. J Immunol 2000, 164:2629-2634

12. Miller CM, Boulter NR, Ikin RJ, Smith NC: The immunobiology of the innate response to Toxoplasma gondii. Int J Parasitol 2009, 39:23-39.

13. Iwasaki A, Medzhitov R: Toll-like receptor control of the adaptive immune responses. Nat Immunol 2004, 5:987-995.

14. Ferreira IM, Vidal JE, Costa-Silva TA, Meira CS, Hiramoto RM, de Penalva Oliveira AC, Pereira-Chioccola VL: Toxoplasma gondii: genotyping of strains from Brazilian AIDS patients with cerebral toxoplasmosis by multilocus PCR-RFLP markers. Exp Parasitol 2008, 118:221-227.

15. Schena M, Shalon D, Davis RW, Brown PO: Quantitative monitoring of gene expression patterns with a complementary DNA microarray. Science 1995, 270:467-470

16. Wu Z, Zhang W, Lu Y, Lu C: Transcriptome profiling of zebrafish infected with Streptococcus suis. Microb Pathog 2010, 48:178-187.

17. Wu JQ, Dwyer DE, Dyer WB, Yang YH, Wang B, Saksena NK: Genome-wide analysis of primary CD4+ and CD8+ T cell transcriptomes shows evidence for a network of enriched pathways associated with HIV disease. Retrovirology 2011, 8:18.

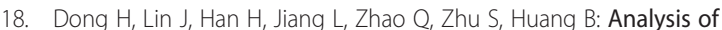
differentially expressed genes in the precocious line of Eimeria maxima and its parent strain using suppression subtractive hybridization and cDNA microarrays. Parasitol Res 2011, 108:1033-1040.

19. Bozdech Z, Mok S, Gupta AP: DNA microarray-based genome-wide analyses of Plasmodium parasites. Methods Mol Biol 2013, 923:189-211.

20. Blader IJ, Manger ID, Boothroyd JC: Microarray analysis reveals previously unknown changes in Toxoplasma gondii-infected human cells. J Biol Chem 2001, 276:24223-24231.

21. Chaussabel D, Semnani RT, McDowell MA, Sacks D, Sher A, Nutman TB: Unique gene expression profiles of human macrophages and dendritic cells to phylogenetically distinct parasites. Blood 2003, 102:672-681.

22. Knight BC, Kissane S, Falciani F, Salmon M, Stanford MR, Wallace GR: Expression analysis of immune response genes of Muller cells infected with Toxoplasma gondii. J Neuroimmunol 2006, 179:126-131.

23. Knight BC, Brunton CL, Modi NC, Wallace GR, Stanford MR: The effect of Toxoplasma gondii infection on expression of chemokines by rat retinal vascular endothelial cells. J Neuroimmuno/ 2005, 160:41-47.

24. Fouts AE, Boothroyd JC: Infection with Toxoplasma gondii bradyzoites has a diminished impact on host transcript levels relative to tachyzoite infection. Infect Immun 2007, 75:634-642.

25. Xiao J, Jones-Brando L, Talbot CC Jr, Yolken RH: Differential effects of three canonical Toxoplasma strains on gene expression in human neuroepithelial cells. Infect Immun 2011, 79:1363-1373.

26. Webster JP: The effect of Toxoplasma gondii on animal behavior: playing cat and mouse. Schizophr Bull 2007, 33:752-756
27. Henriquez SA, Brett R, Alexander J, Pratt J, Roberts CW: Neuropsychiatric disease and Toxoplasma gondii infection. Neuroimmunomodulation 2009 16:122-133.

28. Suzuki Y: Host resistance in the brain against Toxoplasma gondii. J Infect Dis 2002, 185(Suppl 1):S58-S65

29. Caggana M, Conroy JM, Pass KA: Rapid, efficient method for multiplex amplification from filter paper. Hum Mutat 1998, 11:404-409.

30. Homan WL, Vercammen M, De Braekeleer J, Verschueren H: Identification of a 200 - to 300 -fold repetitive 529 bp DNA fragment in Toxoplasma gondii, and its use for diagnostic and quantitative PCR. Int J Parasitol 2000, 30:69-75.

31. Wang J, Liu X, Jia B, Lu H, Peng S, Piao X, Hou N, Cai P, Yin J, Jiang N, Chen Q: A comparative study of small RNAs in Toxoplasma gondii of distinct genotypes. Parasit Vectors 2012, 5:186.

32. Gene Ontology Consortium: The gene ontology (GO) project in 2006. Nucleic Acids Res 2006, 34:D322-D326.

33. Ashburner M, Ball CA, Blake JA, Botstein D, Butler H, Cherry JM, Davis AP, Dolinski K, Dwight SS, Eppig JT, et al: Gene ontology: tool for the unification of biology. The gene ontology consortium. Nat Genet 2000 25:25-29.

34. Myhre S, Tveit H, Mollestad T, Laegreid A: Additional gene ontology structure for improved biological reasoning. Bioinformatics 2006, 22:2020-2027.

35. Dupuy D, Bertin N, Hidalgo CA, Venkatesan K, Tu D, Lee D, Rosenberg J, Svrzikapa N, Blanc A, Carnec A, et al: Genome-scale analysis of in vivo spatiotemporal promoter activity in Caenorhabditis elegans. Nat Biotechnol 2007, 25:663-668.

36. Kanehisa M, Goto S, Kawashima S, Okuno Y, Hattori M: The KEGG resource for deciphering the genome. Nucleic Acids Res 2004, 32:D277-D280.

37. McHale CM, Zhang L, Lan Q, Li G, Hubbard AE, Forrest MS, Vermeulen R, Chen J, Shen M, Rappaport SM, et al: Changes in the peripheral blood transcriptome associated with occupational benzene exposure identified by cross-comparison on two microarray platforms. Genomics 2009, 93:343-349

38. Yi M, Horton JD, Cohen JC, Hobbs HH, Stephens RM: WholePathwayScope: a comprehensive pathway-based analysis tool for high-throughput data. BMC Bioinforma 2006, 7:30

39. Livak KJ, Schmittgen TD: Analysis of relative gene expression data using real-time quantitative PCR and the 2(-Delta Delta C(T)) Method. Methods 2001, 25:402-408

40. Torrey EF, Yolken RH: Toxoplasma gondii and schizophrenia. Emerg Infect Dis 2003, 9:1375-1380

41. Carruthers VB, Suzuki Y: Effects of Toxoplasma gondii infection on the brain. Schizophr Bull 2007, 33:745-751

42. Zhou DH, Zhao FR, Huang SY, Xu MJ, Song HQ, Su C, Zhu XQ: Changes in the proteomic profiles of mouse brain after infection with cyst-forming Toxoplasma gondii. Parasit Vectors 2013, 6:96.

43. Braak H, Braak E: Neuropathological stageing of Alzheimer-related changes. Acta Neuropathol 1991, 82:239-259.

44. Kusbeci OY, Miman O, Yaman M, Aktepe OC, Yazar S: Could Toxoplasma gondii have any role in Alzheimer disease? Alzheimer Dis Assoc Disord 2011, 25:1-3.

45. Jung BK, Pyo KH, Shin KY, Hwang YS, Lim H, Lee SJ, Moon JH, Lee SH, Suh $\mathrm{YH}$, Chai JY, Shin EH: Toxoplasma gondii infection in the brain inhibits neuronal degeneration and learning and memory impairments in a murine model of Alzheimer's disease. PLoS One 2012, 7:e33312.

46. Castillo-Quan Jl: Rosiglitazone effects to ameliorate Alzheimer's disease pathogenic features: insulin signaling and neurotrophic factors. J Neuropsychiatry Clin Neurosci 2009, 21:347-348.

47. Clodfelder-Miller BJ, Zmijewska AA, Johnson GV, Jope RS: Tau is hyperphosphorylated at multiple sites in mouse brain in vivo after streptozotocin-induced insulin deficiency. Diabetes 2006, 55:3320-3325.

48. Yarovinsky F, Zhang D, Andersen JF, Bannenberg GL, Serhan CN, Hayden MS, Hieny S, Sutterwala FS, Flavell RA, Ghosh S, Sher A: TLR11 activation of dendritic cells by a protozoan profilin-like protein. Science 2005, 308:1626-1629.

49. Scanga CA, Aliberti J, Jankovic D, Tilloy F, Bennouna S, Denkers EY, Medzhitov R, Sher A: Cutting edge: MyD88 is required for resistance to Toxoplasma gondii infection and regulates parasite-induced IL-12 production by dendritic cells. J Immunol 2002, 168:5997-6001.

50. Khan IA, Ely KH, Kasper LH: Antigen-specific CD8+ T cell clone protects against acute Toxoplasma gondii infection in mice. J Immunol 1994, 152:1856-1860 
51. Lin SS, Gross U, Bohne W: Type II NADH dehydrogenase inhibitor 1hydroxy-2-dodecyl-4(1H)quinolone leads to collapse of mitochondrial inner-membrane potential and ATP depletion in Toxoplasma gondii. Eukaryot Cell 2009, 8:877-887.

52. Weilhammer DR, lavarone AT, Villegas EN, Brooks GA, Sinai AP, Sha WC: Host metabolism regulates growth and differentiation of Toxoplasma gondii. Int J Parasitol 2012, 42:947-959.

doi:10.1186/1756-3305-6-161

Cite this article as: Jia et al:: Genome-wide comparative analysis

revealed significant transcriptome changes in mice after Toxoplasma gondii infection. Parasites \& Vectors 2013 6:161

\section{Submit your next manuscript to BioMed Central and take full advantage of:}

- Convenient online submission

- Thorough peer review

- No space constraints or color figure charges

- Immediate publication on acceptance

- Inclusion in PubMed, CAS, Scopus and Google Scholar

- Research which is freely available for redistribution 